\section{Emotional or educational debriefing after}

\section{psychological trauma*}

\author{
Randomised controlled trial \\ MARIT SIJBR ANDIJ, MIR ANDA OLFF, JOHANNES B. REITSMA, \\ INGRID V. E. CARLIER and BERTHOLD P. R. GERSONS
}

\begin{abstract}
Background Recent studies show that individual single-session psychological debriefing does not prevent and can even aggravate symptoms of post-traumatic stress disorder (PTSD).
\end{abstract}

\section{Aims We studied the effect of emotional ventilation debriefing and educational debriefing $v$. no debriefing on symptoms of PTSD, anxiety and depression.}

\begin{abstract}
Method We randomised 236 adult survivors of a recent traumatic event to either emotional ventilation debriefing, educational debriefing or no debriefing (control) and followed up at 2 weeks, 6 weeks and 6 months.
\end{abstract}

\section{Results Psychiatric symptoms decreased in all three groups over time, without significant differences between the groups in symptoms of PTSD $(P=0.33)$. Participants in the emotional debriefing group with high baseline hyperarousal score had significantly more PTSD symptoms at 6 weeks than control participants $(P=0.005)$.}

\section{Conclusions Our study did not provide evidence for the usefulness of individual psychological debriefing in reducing symptoms of PTSD, anxiety and depression after psychological trauma.}

Declaration of interest None. Funding detailed in Acknowledgements.

*Preliminary results were presented to the International Society of Traumatic Stress Studies at their 18th and 21st annual conferences.
Although single-session psychological debriefing is offered as immediate psychological assistance to survivors of all kinds of traumatic events, its efficacy in the prevention of symptoms of post-traumatic stress disorder (PTSD), anxiety or depression is not empirically supported (Litz et al, 2002; Rose et al, 2002; van Emmerik et $a l, 2002)$. Some studies even indicate adverse effects (Bisson et al, 1997; Mayou et al, 2000), which have been explained in several ways. It has been argued that the stimulation of emotional ventilation soon after a traumatic event may be too overwhelming for some survivors, whereas a period of rest and reduced talking about the event may in fact be an adaptive response (Ursano et al, 2000). Furthermore, the psychoeducation provided during the debriefing may increase the awareness of stress symptoms that would otherwise not have been noted (Raphael \& Meldrum, 1995), or 'change heroes into patients'. The effects of the constitutive elements of debriefing, i.e. emotional ventilation and psychoeducation, have never been systematically studied. The present randomised controlled trial was designed to assess the efficacy of individual single-session debriefing based on emotional ventilation alone or psychoeducation alone in preventing symptoms of PTSD in relation to a control group that had no debriefing. A secondary question was whether symptoms of acute psychological distress interact with the effect of each debriefing method.

\section{METHOD}

\section{Participants and design}

The study was conducted at the Centre for Psychological Trauma at the Academic Medical Centre in Amsterdam, The Netherlands, which is an out-patient clinic for diagnosis and treatment of people with trauma-related psychiatric disorders. Participants were civilian trauma survivors who were referred by the Emergency Department and Trauma Unit, victim support workers, general practitioners and company doctors in the Amsterdam area. Recruitment took place from December 1999 to November 2001; collection of follow-up data finished in May 2002.

Inclusion criteria were: (a) having experienced a single traumatic event fulfilling the criterion A1 of the diagnosis of PTSD in the DSM-IV (American Psychiatric Association, 2001); (b) traumatic event occurred less than 2 weeks previously; (c) age 18 years or older; (d) proficiency in Dutch.

Exclusion criteria were: (a) suicidal ideation; (b) already having received a debriefing session since the trauma.

Sample size calculations suggested that each group should consist of 64 participants to detect a medium effect size $(d=0.5)$ with a power of $80 \%$ and a twosided significance level of $5 \%$ (Cohen, 1977). To allow for sample attrition we decided to enrol at least 225 participants (75 participants in each group) during the 2-year inclusion period. We assigned participants randomly to one of three groups: emotional debriefing, educational debriefing or no debriefing (control). Randomisation was carried out on a 1:1:1 basis using block sizes that randomly varied between six and nine participants, and was performed by the principal investigator (M.S.) on a central computer, and a log file of all randomisations was kept. Participants were not masked to their intervention, but they were asked not to reveal this information to the research assistants who conducted the assessments, as these assistants were masked to the allocated interventions.

Participants were invited to four assessments: a pre-intervention assessment (baseline) and three follow-up assessments: at 2 weeks, 6 weeks and 6 months after the intervention. Written informed consent was obtained from all participants after full description of the study protocol. The study protocol was approved by the Medical Ethics Committee of the Academic Medical Centre.

\section{Interventions}

Approximately 2 weeks after experiencing the traumatic incident (median 15 days, range 11-19), participants received either the emotional debriefing, psychoeducational debriefing or no debriefing (control). We based the 2-week interval between trauma and debriefing on medical ethical 
considerations, as it was then assumed that an early timing of the intervention contributed to the harmful effect (Chemtob et al, 1997). The emotional and educational debriefings were based on the Critical Incidents Stress Debriefing protocol originally designed by Mitchell (CISD; Mitchell, 1983; Mitchell \& Everly, 2001), but with exclusion of the psychoeducational elements and the emotional elements respectively.

Emotional debriefing consisted of five stages:

(1) Introduction (explaining goals of the session)

(2) Facts (participants describe the facts of the trauma as they see them)

(3) Thought (participants recall their first thoughts during the trauma)

(4) Reaction (participants reconstruct the trauma and accompanying emotions in detail)

(7) Re-entry (information about other services available and closure of the session).

Two stages were excluded:

(5) Symptoms (participants describe stress symptoms they experienced during, just after the event and currently)

(6) Teaching stage (information about stress symptoms and post-traumatic stress symptoms, and tips and advice about ways of coping with the trauma or the stress symptoms).

Educational debriefing consisted of six stages of the Mitchell protocol:

(1) Introduction

(2) Facts

(3) Thought

(5) Symptoms

(6) Teaching

(7) Re-entry.

The Reaction stage (4) was excluded. Both types of debriefing lasted $45 \mathrm{~min}$ to $1 \mathrm{~h}$ and were individually administered. Eight clinical psychologists performed the debriefing; these were trained during 2 days by the authors (I.C. and B.G.) in administering the debriefing protocols. Protocol adherence was ensured by monthly supervision, and was measured by a rating system specifically designed for this study. In this rating system, we measured the occurrence of both desired and undesired components in audiotaped sessions of both types of debriefing, following the recommendations of Waltz et al (1993). The rating system consisted of three parts, i.e. general, proscribed and forbidden behaviours, which were combined in an overall protocol adherence score. Raters were nine clinical psychologists. A random sample of 43 briefings was independently scored by two raters. Interrater reliability was good, with an intraclass correlation coefficient of 0.77 (95\% CI $0.58-0.88)$. According to the raters, $88 \%$ (range $67 \%-100 \%$ ) of the desired protocol components occurred.

\section{Measures}

Severity of symptoms of PTSD, anxiety and depression was assessed at baseline (preintervention assessment) and at all three follow-up assessments ( 2 weeks, 6 weeks and 6 months after the intervention). Nine clinical psychologists conducted the assessments. All assessments of one participant were performed by the same person.

Symptoms were measured with the Structured Interview for PTSD (SI-PTSD; Carlier et al, 1998; Davidson et al, 1989), which is a 17-item clinical interview that records the presence and severity of the 17 DSM-IV diagnostic criteria for PTSD. Each item is rated on a $0-4$ scale; scores of 3 or higher indicate the presence of that particular symptom. In accordance with DSM-IV, interview items are clustered into the three PTSD symptom groups: re-experiencing (5 symptoms), avoidance (7 symptoms) and hyperarousal ( 5 symptoms). In the presence of at least one re-experiencing symptom, at least three avoidance symptoms and at least two hyperarousal symptoms during 1 month, PTSD according to DSM-IV may be diagnosed. The sum of the item scores results in a maximum continuous PTSD score of 68 . Higher scores indicate the presence of more symptoms. In this study, we also used the baseline SI-PTSD scores to measure acute psychological distress. For that purpose, re-experiencing, avoidance and hyperarousal scores were dichotomised into high and low using the cut-offs for DSM-IV diagnosis. SI-PTSD scores correlate highly with clinicians' ratings and with other similar self-report PTSD instruments (Carlier et al, 1998; Davidson et al, 1989). For the Dutch version of the SI-PTSD, adequate internal consistency (Cronbach's $\alpha=0.93$ ) and interrater reliability were found (Cohen's $\kappa=0.88$; Carlier et al, 1998).

States of anxiety and depression were measured with the Hospital Anxiety and Depression Scale (HADS; Zigmond \&
Snaith, 1983; Spinhoven et al, 1997), a well-established 14-item scale consisting of two sub-scales: HADS-A (anxiety, 7 items, range 0-21) and HADS-D (depression, 7 items, range 0-21). Higher scores indicate more anxiety and/or depression. The Dutch version of the HADS showed satisfactory reliability and validity (Spinhoven $e t$ al, 1997).

\section{Data analysis}

We used chi-squared tests and independent $t$-tests to examine whether participants lost to follow-up differed from other participants. For the main outcomes, we used repeated-measurement analyses to study the changes over time in SI-PTSD and HADS scores between the three intervention groups. We applied mixed linear models to take into account that measurements within the same individual are correlated (Verbeke \& Molenberghs, 1997). No mathematical pattern was imposed on the covariance structure for measurements within the same individual (unstructured). Another advantage of this repeated measurements model is that not only the complete cases, but all available cases, are used in the analysis. The mean score for each outcome was modelled as a function of the intervention given (three levels), time since intervention (as a categorical variable with three levels) and the pre-intervention measurement (continuous). The interaction term between time and intervention was added to the model to test whether trends over time differed for the three intervention groups. To determine whether symptoms of acute psychological distress influence the effect of the intervention, we added the following interaction terms to the model: re-experiencing, avoidance and hyperarousal at baseline (all dichotomised into high and low).

All our analyses were on an intentionto-treat basis, unless otherwise indicated. A two-tailed $\alpha$ level of $P=0.05$ was used to determine statistical significance. For all analyses, the Statistical Package for the Social Sciences, version 11.0.1 for Windows was used.

\section{RESULTS}

\section{Participants}

Of the 295 respondents who were assessed for eligibility, 236 were randomised (76 to emotional, 79 to educational and 81 to no debriefing). Another 59 respondents were 
excluded, because they fulfilled criteria for any of the DSM-IV disorders specified in the exclusion criteria $(n=10,16.9 \%)$, the trauma was ongoing $(n=4,6.8 \%)$, they had not mastered the Dutch language $(n=5,8.5 \%)$, they had already received a debriefing $(n=1,1.7 \%)$, they refused $(n=35,59.3 \%)$ or other reasons $(n=4$, $6.8 \%)$. The numbers of participants who were lost to the 2 weeks' ( $n=43,18.2 \%)$, 6 weeks' $((n=47,19.9 \%)$ and 6 months' $(n=59,25.0 \%)$ follow-up were equally distributed across the study groups; 35 participants $(14.8 \%)$ missed all three follow-up assessments, $11(4.7 \%)$ missed two assessments and $22(9.3 \%)$ missed one assessment. Finally, 12 participants $(5.1 \%$; 3 in emotional and 9 in educational debriefing) did not receive the allocated debriefing. These participants were excluded from the completers' analysis (Fig. 1).

Baseline characteristics are presented in Table 1. Chi-squared tests and independent $t$-tests showed that significantly more participants in the control group were employed than in the other two groups $\left(\chi^{2}=6.2\right.$, d.f. $=2, P=0.046)$, more participants in educational debriefing had secondary school as their highest education $\left(\chi^{2}=11.7\right.$, d.f. $\left.=4, P=0.020\right)$ or experienced an accident rather than an assault $\left(\chi^{2}=6.6\right.$, d.f. $=2, P=0.04)$ than in the other two groups. No other significant differences in baseline characteristics between the study groups were found.

\section{Main outcomes}

The mean SI-PTSD and HADS anxiety and depression scores at the three follow-up assessments are shown in Table 2.

Mixed-model analysis on SI-PTSD total scores based on all 236 participants showed that the severity of PTSD decreased over time in all three groups $(P<0.001)$, but that there was no significant difference in SI-PTSD total score between groups $(F=1.17$, d.f. $=174, P=0.33$ ) (Fig. 2). The estimated reductions for the SI-PTSD between 2 weeks' and 6 months' follow-up (adjusted for baseline) were 7.1 in the emotional (95\% CI 4.7-9.5), 6.4 in the educational $(95 \%$ CI $4.0-8.8)$ and 5.9 in the no debriefing group (95\% CI 3.6-8.2). No significant differences between intervention groups were found on the SI-PTSD subscales of re-experiencing $(P=0.058)$, avoidance $(P=0.84)$ or hyperarousal $(P=0.20)$. Completer analysis of SI-PTSD scores in which the 12 participants who did not

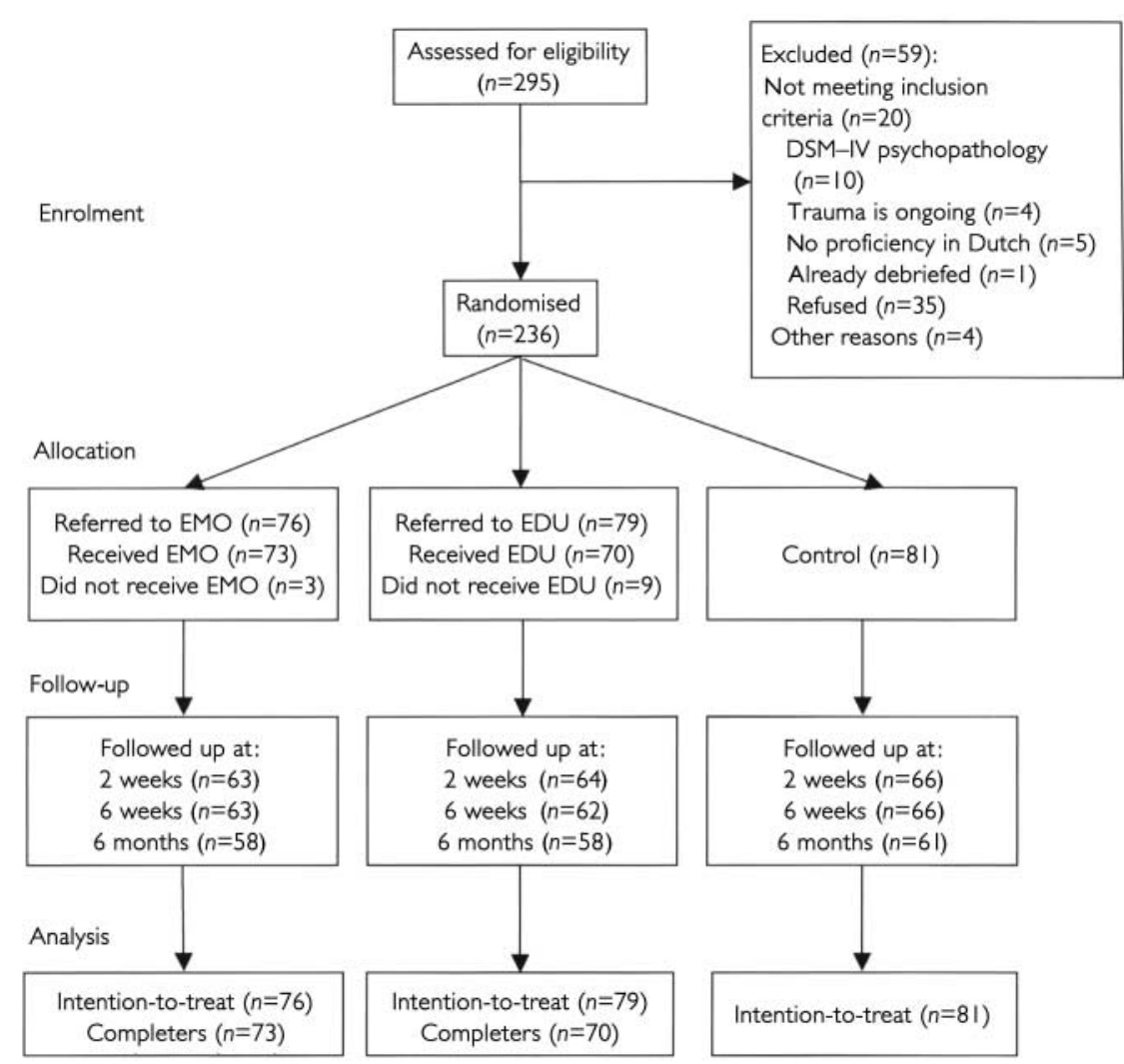

Fig. I Study protocol and flow of patients through trial. EMO, emotional debriefing; EDU, educational debriefing.

Table I Baseline characteristics of the study group $(n=236)$

\begin{tabular}{|c|c|c|c|}
\hline Characteristics & $\begin{array}{c}\text { Emotional } \\
\text { debriefing } \\
(n=76)\end{array}$ & $\begin{array}{l}\text { Educational } \\
\text { debriefing } \\
\quad(n=79)\end{array}$ & $\begin{array}{l}\text { Control } \\
(n=8 \mathrm{I})\end{array}$ \\
\hline Age, years: mean (s.d.) & $41.7(12.3)$ & $38.3(12.8)$ & $41.2(13.6)$ \\
\hline Male gender, $n(\%)$ & $40(52.6)$ & $36(45.6)$ & $45(55.6)$ \\
\hline Employed, $n(\%)^{\prime}$ & $18(25.7)$ & $22(31.0)$ & $35(44.3)$ \\
\hline \multicolumn{4}{|l|}{ Education, $n(\%)^{2}$} \\
\hline Primary school & $22(31.4)$ & $8(I I .4)$ & $22(28.2)$ \\
\hline Secondary school & $34(48.6)$ & $4 I(58.6)$ & $31(39.7)$ \\
\hline Postgraduate & $14(20.0)$ & $21(30.0)$ & $25(32.1)$ \\
\hline Dutch ethnicity, $n(\%)^{3}$ & $59(84.3)$ & $57(80.3)$ & $65(83.3)$ \\
\hline \multicolumn{4}{|l|}{ Type of trauma, $n(\%)$} \\
\hline Assault & $46(60.5)$ & $32(40.5)$ & $44(54.3)$ \\
\hline Accident & $30(39.5)$ & $47(59.5)$ & $37(45.7)$ \\
\hline PTSD score (SI-PTSD): mean (s.d.) & $19.9(12.2)$ & $19.9(12.7)$ & I7.7 (II.0) \\
\hline Anxiety score (HADS): mean (s.d.) ${ }^{4}$ & $8.8(6.0)$ & $8.6(5.7)$ & $8.4(5.6)$ \\
\hline Depression score (HADS): mean (s.d.) ${ }^{4}$ & $7.1(5.7)$ & $6.8(5.9)$ & $6.6(5.5)$ \\
\hline
\end{tabular}

PTSD, post-traumatic stress disorder; SI-PTSD, Structured Interview for PTSD; HADS, Hospital Anxiety and Depression Scale.

I. Data from 220 participants.

2. Data from 218 participants.

3. Data from 219 participants.

4. Data from 223 participants. 
Table 2 Main outcome measures $(n=236)$

\begin{tabular}{|c|c|c|c|c|c|c|}
\hline \multirow{2}{*}{$\begin{array}{l}\text { Psychopathology } \\
\text { measure }\end{array}$} & \multicolumn{2}{|c|}{ Emotional debriefing } & \multicolumn{2}{|c|}{ Educational debriefing } & \multicolumn{2}{|c|}{ Control } \\
\hline & $n$ & Mean (s.d.) & $n$ & Mean (s.d.) & $n$ & Mean (s.d.) \\
\hline \multicolumn{7}{|l|}{ SI-PTSD } \\
\hline 2 weeks & 63 & I8.I (13.2) & 63 & $16.2(10.7)$ & 63 & $15.9(10.9)$ \\
\hline 6 weeks & 60 & $14.4(13.8)$ & 60 & $11.9(11.7)$ & 65 & $10.5(9.1)$ \\
\hline 6 months & 55 & $10.2(12.0)$ & 55 & $9.3(9.4)$ & 59 & $9.6(10.1)$ \\
\hline \multicolumn{7}{|l|}{ Anxiety (HADS) } \\
\hline 2 weeks & 62 & $7.6(6.0)$ & 63 & $6.6(5.0)$ & 65 & $6.4(5.0)$ \\
\hline 6 weeks & 63 & $5.6(5.2)$ & 61 & $5.1(5.0)$ & 66 & $4.7(4.6)$ \\
\hline 6 months & 58 & $5.0(5.2)$ & 57 & $4.4(4.0)$ & 61 & $4.6(4.7)$ \\
\hline \multicolumn{7}{|l|}{ Depression } \\
\hline \multicolumn{7}{|l|}{ (HADS) } \\
\hline 2 weeks & 62 & $5.7(5.4)$ & 62 & $4.7(4.6)$ & 64 & $4.5(4.9)$ \\
\hline 6 weeks & 63 & $4.3(4.8)$ & 61 & $3.3(4.2)$ & 66 & $3.7(4.5)$ \\
\hline 6 months & 58 & $3.8(4.8)$ & 57 & $3.2(4.0)$ & 60 & $3.2(4.1)$ \\
\hline
\end{tabular}

PTSD, post-traumatic stress disorder; SI-PTSD, Structured Interview for PTSD; HADS, Hospital Anxiety and Depression Scale.

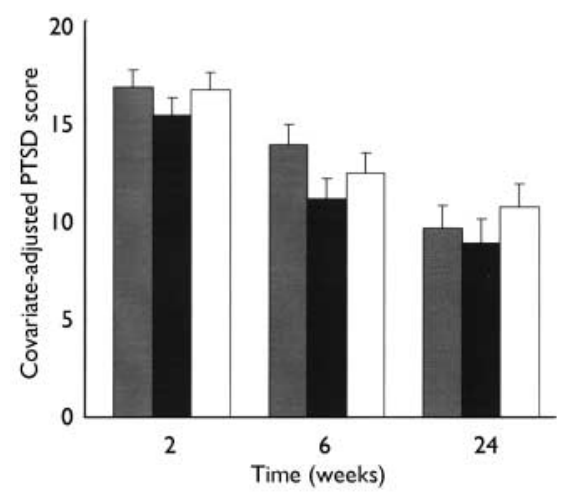

Fig. 2 Post-traumatic stress disorder (PTSD) scores as measured by Structured Interview for PTSD in participants $(n=236)$ randomly assigned to an emotional or educational debriefing or a waitinglist control condition. Mean (s.e.) values at baseline, 2 weeks, 6 weeks and 6 months from a repeatedmeasurement model adjusting for baseline value of PTSD score. $\square$, Emotional debriefing;

, educational debriefing; $\square$, no debriefing.

receive the allocated debriefing were excluded revealed similar results, showing no significant differences between groups in SI-PTSD total score $(P=0.28)$, reexperiencing $(P=0.058)$, avoidance $(P=$ $0.82)$ or hyperarousal score $(P=0.15)$.

Mixed-model analysis based on all 236 participants showed that HADS anxiety scores decreased significantly over time in all three groups $(P<0.001)$, without a significant difference between intervention groups $(F=0.15$, d.f. $=175, P=0.96)$. The mean reductions in HADS anxiety scores between 2 weeks' and 6 months' followup (adjusted for baseline) were estimated 2.2 in the educational $(95 \%$ CI $1.2-3.2)$ and 2.1 in the no debriefing groups $(95 \%$ CI 1.1-3.0). HADS depression score also decreased over time in all three groups $(P<0.001)$, without a significant difference between intervention groups $(F=1.4$, d.f. $=175, P=0.23)$. The mean reductions in HADS depression scores between 2 weeks' and 6 months' follow-up (adjusted for baseline) were estimated as 1.6 in the emotional $(95 \%$ CI $0.6-2.6), 1.5$ in the educational (95\% CI $0.5-2.5)$ and 1.4 in Completer analyses were consistent with intention-to-treat results, showing no significant differences between groups in HADS anxiety $(P=0.95)$ or depression scores $(P=0.20)$.

At baseline, a total of 23 participants $(9.7 \%)$ fulfilled the diagnostic criteria for PTSD, ignoring the time criterion. At 2 weeks' follow-up, the disorder was diagnosed in 10 participants $(5.4 \%)$, at 6 weeks' follow-up in 9 participants $(4.9 \%)$ and at 6 months' follow up in 8 participants $(4.8 \%)$. No significant differences between the three intervention groups in the distribution of participants with and without the diagnosis were found.

\section{Subgroup analyses}

To examine whether in this study the effect of an intervention interacted with acute as 2.4 in the emotional (95\% CI 1.4-3.3), the no debriefing group (95\% CI 0.4-2.4). psychological distress, we added the following factors to our model: high $v$. low intrusion, avoidance, and hyperarousal at baseline. Based on cut-off scores of one symptom present for intrusion, three for avoidance and two for hyperarousal, 147 participants $(62.3 \%)$ had high intrusion, 29 participants $(12.2 \%)$ had high avoidance and 59 (25.0\%) had high hyperarousal. Mixed-model analyses based on all 236 participants showed that effects of debriefing were not different in any of these subgroups, with the exception of the subgroup of participants with two or more hyperarousal symptoms. Participants in the emotional debriefing group with two or more hyperarousal symptoms had significantly higher PTSD scores than similar participants in the control group at 6 weeks after the intervention (test for interaction $P=0.005$ for SI-PTSD score). There were no other differences between groups. Subgroup analyses based on completers were consistent with those of the intention-totreat analysis and did not show a differential effect for debriefing in any of the subgroups as defined above, with the exception of the subgroup of participants with two or more hyperarousal symptoms at baseline. These participants had significantly higher PTSD scores if they had received emotional debriefing than similar participants in the control group at the 6 weeks' follow-up (test for interaction $P=0.003$ for SI-PTSD score).

\section{DISCUSSION}

The main goal of this randomised controlled trial was to study the effect of two adaptations of the usual debriefing protocol, i.e. emotional debriefing or educational debriefing in relation to a control group that received no debriefing. The results show that in all groups symptoms decreased significantly over the 6-month period, without any differences between the two debriefing methods and no debriefing. In addition, emotional debriefing had an adverse effect in participants with early hyperarousal symptoms, in that participants with two or more of the five hyperarousal symptoms had higher PTSD scores 6 weeks after an emotional debriefing session than similar participants in the control group.

\section{Relation of findings to previous debriefing studies}

The absence of an effect of debriefing in our overall study group is in line with the 
results of recent randomised clinical trials in which no differences were found between debriefed trauma victims and nondebriefed victims in symptoms of PTSD, anxiety or depression (Conlon et al, 1999; Rose et al, 1999), but differs from individual debriefing trials that showed adverse effects (Hobbs et al, 1996; Bisson et al, 1997). A difference between our study and previous studies is that we included a relatively heterogeneous group of participants with regard to their type of traumatic experience. Also, we found a substantially lower rate of PTSD across the three study groups (mean $5.4 \%$ at 1 month) than was found in earlier studies on debriefing (varying from $19 \%$ at 3 months to $26 \%$ at 6 months after the traumatic event; Conlon et al, 1999; Rose et al, 1999). The low occurrence of PTSD in our trial was not anticipated; rather, we expected that our participants would be more likely to be symptomatic because they had been referred. However, within our subgroup of participants with two or more early hyperarousal symptoms, rates and severity of PTSD were very similar to those found in earlier debriefing trials (Hobbs et al, 1996; Bisson et al, 1997; Mayou et al, 2000), which might explain the fact that the adverse effects were limited to that subgroup. Another difference between our study and previous studies is that we found only short-term negative effects in the participants with hyperarousal whereas, in previous studies, long-term adverse effects were found at 13 months (Bisson et al, 1997) or adverse effects were more pronounced at 3 years than at 4 months (Mayou et al, 2000). Possibly the four assessment interviews influenced natural recovery, making the three groups more equal with regard to the attention received at the end-point of our trial.

\section{Role of hyperarousal in response to emotional debriefing}

The possibility that some survivors, especially those with high arousal, are put at heightened risk for adverse outcomes as a result of debriefing was previously assumed by professionals attending a workshop to reach consensus on early interventions following mass violence (National Institute of Mental Health, 2002), an assumption now supported by the subgroup results in this trial. The relationship between high initial hyperarousal and adverse effect of emotional debriefing, after first controlling for baseline PTSD symptoms, could be explained as follows. In previous studies it has been established that high degrees of arousal in the immediate aftermath of a traumatic event are associated with an increased risk for the development of PTSD, measured both by self-report (Carlier et al, 1997; Schell et al, 2004) and physiologically by means of heart rate response (Shalev et al, 1998; Bryant et al, 2000; Zatzick et al, 2005). Encouraging highly aroused trauma survivors to express their feeling and emotions concerning the trauma might activate the sympathetic nervous system to such a degree that successful encoding of the traumatic memory is disrupted. Moreover, during an emotional debriefing session negative appraisal of one's sense of mastery may be promoted (Weisaeth, 2000). This is assumed to keep the hyperreactive individual in a state of high arousal which may cause symptoms of PTSD to escalate rather than resolve (McCleery \& Harvey, 2004).

\section{Strengths and limitations}

Our trial had several methodological strengths. First, we used randomisation to assign participants to intervention groups and masked outcome assessment. Second, protocol adherence was systematically assessed, which to our knowledge has never been done before in debriefing research. Third, intention-to-treat analysis was compared with completer analysis. A limitation might be that the relatively low PTSD rate in our overall study group caused a loss of statistical power, leaving small differences between intervention groups undetected. Another limitation might be the possibility that there was some overlap between the emotional and educational debriefing protocols in their content. In both interventions participants were asked to give a description of the traumatic event (in the 'Facts phase'), so that - even though it was discouraged by the debriefers - participants in the educational debriefing group might have expressed their emotions during that part of the intervention. Furthermore, translating our results to practice should be done with caution. Since we applied debriefing individually, the results cannot be generalised to group settings. Finally, based on medical-ethical considerations we were not allowed to offer the debriefing session until 2 weeks after the traumatic experience, whereas in most instances debriefing is offered within a few days of the trauma.

\section{Clinical and practical implications}

The practice of offering single-session psychological debriefing to trauma victims in order to prevent symptoms of PTSD, anxiety and depression is not supported by the results from this study or earlier research (Litz et al, 2002; van Emmerik et al, 2002; Rose et al, 2002). Our findings are in line with recent expert statements in which the use of single-session individual interventions focusing on the traumatic event or the expression of emotions for all those involved is not recommended (National Institute of Mental Health, 2002; National Collaborating Centre for Mental Health, 2005). The fact that singlesession trauma-focused interventions do not ameliorate psychological distress resulting from traumatic experience, and that the focus on emotions even appears to negatively affect psychological recovery at least in some trauma victims, show that there are all too many reasons for discontinuing its use in practice. On the basis of current evidence, more benefits are expected from early treatment of only those patients with acute stress disorder or acute PTSD with four or five sessions of cognitivebehavioural therapy (Bryant et al, 1998, 1999, 2003; Bisson et al, 2004) or 12 sessions of cognitive therapy (Ehlers et al, 2003) in order to prevent a chronic course of PTSD.

Thus, there is no evidence for the usefulness of individual single-session emotional or educational debriefing in reducing psychiatric symptoms of individuals who have experienced various kinds of traumatic events. Moreover, this study highlighted the contribution of early hyperarousal symptoms to the adverse effects of single-session emotion-focused psychological debriefing.

\section{ACKNOWLEDGEMENTS}

The study was supported by a grant from The Netherlands Organisation for Health Research and Development.

\section{REFERENCES}

American Psychiatric Association (200I) Diagnostic and Statistical Manual of Mental Disorders (4th edn) (DSM-IV). Washington, DC: APA.

Bisson, J. I., Jenkins, P. L., Alexander, J., et al (1997) Randomised controlled trial of psychological debriefing for victims of acute burn trauma. British Journal of Psychiatry, I7I, 78-81.

Bisson, J. I., Shepherd, J. P., Joy, D., et al (2004) Early cognitive-behavioural therapy for post-traumatic stress 
symptoms after physical injury. Randomised controlled trial. British Journal of Psychiatry, 184, 63-69.

Bryant, R. A., Harvey, A. G., Dang, S. T., et al (1998) Treatment of acute stress disorder: a comparison of cognitive-behavioral therapy and supportive counseling. Journal of Consulting and Clinical Psychology, 66, 862-866.

Bryant, R. A., Sackville, T., Dang, S. T., et al (1999) Treating acute stress disorder: an evaluation of cognitive behavior therapy and supportive counseling techniques. American Journal of Psychiatry, 156, 1780-1786.

Bryant, R. A., Harvey, A. G., Guthrie, R. M., et al (2000) A prospective study of psychophysiological arousal, acute stress disorder, and posttraumatic stress disorder. Journal of Abnormal Psychology, 109, 34I-344.

Bryant, R. A., Moulds, M., Guthrie, R., et al (2003) Treating acute stress disorder following mild traumatic brain injury. American Journal of Psychiatry, 160, 585-587.

\section{Carlier, I.V., Lamberts, R. D. \& Gersons, B. P. (1997)} Risk factors for posttraumatic stress symptomatology in police officers: a prospective analysis. Journal of Nervous and Mental Disease, 185, 498-506.

Carlier, I. V., Lamberts, R. D., Van Uchelen, A. J., et al (1998) Clinical utility of a brief diagnostic test for posttraumatic stress disorder. Psychosomatic Medicine, 60, $42-47$.

\section{Chemtob, C. M., Tomas, S., Law, W., et al (1997)} Postdisaster psychosocial intervention: a field study of the impact of debriefing on psychological distress. American Journal of Psychiatry, 154, 415-417.

Cohen, J. (1977) Statistical Power Analysis for the Behavioral Sciences. New York: Academic Press.

Conlon, L., Fahy, T. J. \& Conroy, R. (1999) PTSD in ambulant RTA victims: a randomized controlled trial of debriefing. Journal of Psychosomatic Research, 46, 37-44.

Davidson, J., Smith, R. \& Kudler, H. (1989) Validity and reliability of the DSM-III criteria for posttraumatic stress disorder. Experience with a structured interview. Journal of Nervous and Mental Disease, 177, 336-34I.

Ehlers, A., Clark, D. M., Hackmann, A., et al (2003) A randomized controlled trial of cognitive therapy, a selfhelp booklet, and repeated assessments as early interventions for posttraumatic stress disorder. Archives of General Psychiatry, 60, 1024-1032.

Hobbs, M., Mayou, R., Harrison, B., et al (1996) A randomised controlled trial of psychological debriefing for victims of road traffic accidents. BMJ, 3/3, I438-1439.

Litz, B.T., Gray, M. J., Bryant, R. A., et al (2002) Early intervention for trauma: current status and future directions. Clinical Psychology - Science and Practice, 9, 112-134

Mayou, R. A., Ehlers, A. \& Hobbs, M. (2000)

Psychological debriefing for road traffic accident victims.

MARIT SIJBRANDII, MA, MIRANDA OLFF, PhD, Department of Psychiatry, Academic Medical Centre; JOHANNES B. REITSMA, MD, PhD, Department of Clinical Epidemiology and Biostatistics, Academic Medical Centre, University of Amsterdam, Amsterdam; INGRID V. E. CARLIER, PhD, Centre of Work-Related Mental Disorders, Altrecht Mental Health Care, Utrecht; BERTHOLD P. R. GERSONS, MD, PhD, Department of Psychiatry, Academic Medical Centre, University of Amsterdam, Amsterdam, The Netherlands

Correspondence: Marit Sijbrandij, Academic Medical Centre, Department of Psychiatry, Tafelbergweg 25, II05 BC, Amsterdam, The Netherlands. Tel.: +3I 20 5668783; fax: +3I 20 6919019; email: e.m.sijbrandij@amc.uva.nl

Three-year follow-up of a randomised controlled trial. British Journal of Psychiatry, 176, 589-593.

McCleery, J. M. \& Harvey, A. G. (2004) Integration of psychological and biological approaches to trauma memory: implications for pharmacological prevention of PTSD. Journal of Traumatic Stress, 17, 485-496.

Mitchell, J.T. (1983) When disaster strikes... the critical incident stress debriefing process. Journal of Emergency Medical Services, 8, 36-39.

Mitchell, J.T. \& Everly Jr, G. S. (200I) Critical Incident Stress Debriefing: an Operations Manual for CISD, Defusing and Other Group Crisis Intervention Services. Ellicott City, MD: Chevron Publishing.

National Collaborating Centre for Mental Health (2005) Post-Traumatic Stress Disorder. The Management of PTSD in Adults and Children in Primary and Secondary Care. Leicester \& London: British Psychological Society \& Gaskell.

National Institute of Mental Health (2002) Mental Health and Mass Violence: Evidence-Based Early Psychological Intervention for Victims/Survivors of Mass Violence. A workshop to reach consensus on best practices. Washington, DC: U.S. Government Printing Office. http: //www. nimh. nih. gov/publicat/ massviolence.pdf.

Raphael, B. \& Meldrum, L. (1995) Does debriefing after psychological trauma work? BMJ, 310, 1479-1480.

Rose, S., Brewin, C. R., Andrews, B., et al (1999) A randomized controlled trial of individual psychological debriefing for victims of violent crime. Psychological Medicine, 29, 793-799.

Rose, S., Bisson, J., Churchill, R., et al (2002) Psychological debriefing for preventing posttraumatic stress disorder. Cochrane Database of Systematic Reviews, issue 2. Oxford: Update Software.

Schell, T. L., Marshall, G. N. \& Jaycox, L. H. (2004) All symptoms are not created equal: the prominent role of hyperarousal in the natural course of posttraumatic psychological distress. Journal of Abnormal Psychology, II3, 189-197.
Shalev, A. Y., Sahar, T., Freedman, S., et al (1998) A prospective study of heart rate response following trauma and the subsequent development of posttraumatic stress disorder. Archives of General Psychiatry, 55, 553-559.

Spinhoven, P., Ormel, J., Sloekers, P. P., et al (1997) A validation study of the Hospital Anxiety and Depression Scale (HADS) in different groups of Dutch subjects. Psychological Medicine, 27, 363-370.

Ursano, R. J., Fullerton, C. S., Vance, K., et al (2000) Debriefing: its role in the spectrum of prevention and acute management of psychological trauma. In Psychological Debriefing; Theory, Practice and Evidence (eds B. Raphael \& J. P.Wilson), pp. 32-42. Cambridge: Cambridge University Press.

van Emmerik, A. A., Kamphuis, J. H., Hulsbosch, A. M., et al (2002) Single session debriefing after psychological trauma: a meta-analysis. Lancet, $\mathbf{3 6 0}$, 766-771.

Verbeke, G. \& Molenberghs, G. (1997) Linear Mixed Models in Practice: a SAS-oriented Approach. New York: Springer.

Waltz, J., Addis, M. E., Koerner, K., et al (1993) Testing the integrity of a psychotherapy protocol: assessment of adherence and competence. Journal of Consulting and Clinical Psychology, 61, 620-630.

Weisaeth, L. (2000) Briefing and debriefing: group psychological interventions in acute stressor situations. In Psychological Debriefing; Theory, Practice and Evidence (eds B. Raphael \& J. P.Wilson), pp. 43-57. Cambridge: Cambridge University Press.

Zatzick, D. F., Russo, J., Pitman, R. K., et al (2005) Reevaluating the association between emergency department heart rate and the development of posttraumatic stress disorder: A public health approach. Biological Psychiatry, 57, 91-95.

Zigmond, A. S. \& Snaith, R. P. (1983) The Hospita Anxiety and Depression Scale. Acta Psychiatrica Scandinavica, 67, 361-370. 